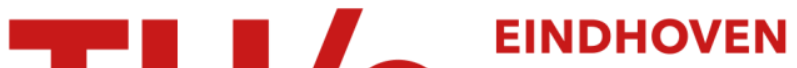 UNIVERSITY OF TECHNOLOGY
}

\section{Numerical simulation of axisymmetric viscous sintering}

\section{Citation for published version (APA):}

van de Vorst, G. A. L. (1994). Numerical simulation of axisymmetric viscous sintering. (RANA : reports on applied and numerical analysis; Vol. 9406). Eindhoven University of Technology.

\section{Document status and date:}

Published: 01/01/1994

\section{Document Version:}

Publisher's PDF, also known as Version of Record (includes final page, issue and volume numbers)

\section{Please check the document version of this publication:}

- A submitted manuscript is the version of the article upon submission and before peer-review. There can be important differences between the submitted version and the official published version of record. People interested in the research are advised to contact the author for the final version of the publication, or visit the $\mathrm{DOI}$ to the publisher's website.

- The final author version and the galley proof are versions of the publication after peer review.

- The final published version features the final layout of the paper including the volume, issue and page numbers.

Link to publication

\section{General rights}

Copyright and moral rights for the publications made accessible in the public portal are retained by the authors and/or other copyright owners and it is a condition of accessing publications that users recognise and abide by the legal requirements associated with these rights.

- Users may download and print one copy of any publication from the public portal for the purpose of private study or research.

- You may not further distribute the material or use it for any profit-making activity or commercial gain

- You may freely distribute the URL identifying the publication in the public portal.

If the publication is distributed under the terms of Article $25 \mathrm{fa}$ of the Dutch Copyright Act, indicated by the "Taverne" license above, please follow below link for the End User Agreement:

www.tue.nl/taverne

Take down policy

If you believe that this document breaches copyright please contact us at:

openaccess@tue.nl

providing details and we will investigate your claim. 
EINDHOVEN UNIVERSITY OF TECHNOLOGY

Department of Mathematics and Computing Science

RANA 94-06

May 1994

Numerical Simulation

of Axisymmetric

Viscous Sintering

by

G.A.L. van de Vorst

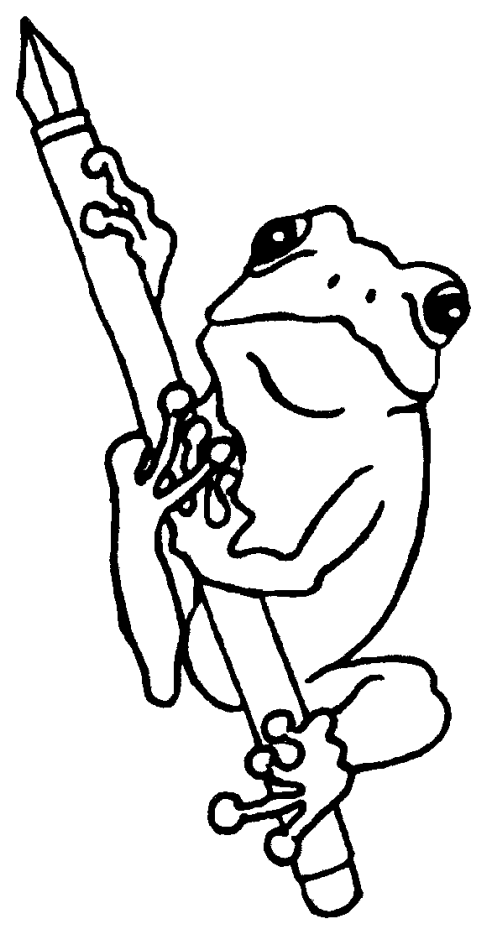


Reports on Applied and Numerical Analysis

Department of Mathematics and Computing Science Eindhoven University of Technology

P.O. Box 513

$5600 \mathrm{MB}$ Eindhoven

The Netherlands

ISSN: 0926-4507 


\title{
Numerical Simulation of Axisymmetric Viscous Sintering
}

\author{
G.A.L. van de Vorst \\ Department of Mathematics and Computing Science \\ Eindhoven University of Technology \\ P.O. Box 513, 5600 MB Eindhoven, The Netherlands \\ (e-mail:wsangv@win.tue.nl)
}

\begin{abstract}
In this paper we describe a numerical method to simulate particular axisymmetric viscous sintering problems. In these problems the material transport is modelled as a viscous incompressible Newtonian volume flow driven solely by surface tension. The numerical simulation is carried out by solving the governing Stokes equations for a fixed domain through a Boundary Element Method (BEM). The resulting velocity field then determines an approximate geometry at a next time level by employing a variable step, variable order Backward Differences Formulae (BDF) method. This numerical algorithm is demonstrated for several simply connected sintering domains, including two coalescing spheres. Furthermore, by considering the movement of a particular fluid region some discrepancies between modelling it as a two-dimensional fluid and as an axisymmetric problem are demonstrated.
\end{abstract}

A.M.S. Classifications: $65 \mathrm{R} 99,76 \mathrm{D} 07$

Keywords $\quad$ : Sintering, Viscosity, Boundary Element Method, Moving Boundaries. 


\section{Introduction}

A method to produce glass fibres for the telecommunication industry is heating a porous pure glass to a sufficiently high temperature so that the glass becomes a highly viscous fluid: the flow causes densification of the glass. The driving force for this phenomenon is the excess of free surface energy of the porous glass compared to a same quantity of a fully dense glass. This process is usually referred to as viscous sintering.

Ideally, one wants to produce a dense and homogeneous glass, free from voids and impurities this way. Therefore, a good theoretical understanding is needed of the densification kinetics of the porous glass, i.e. the viscous sintering phenomenon. In particular, one is interested in the shrinkage rate of the glass as a function of the viscosity and particle size, which reflects how time, temperature and microstructure influence the development of the densification process. Another question is what kind of structural configuration leads to a higher densification rate.

A simple approach of describing the sintering phenomenon is to consider the behaviour of simple systems only, like the coalescence of two spheres, which can be used to understand the behaviour of macroscopic systems. A more sophisticated approach is the determination of a representative unit cell within the porous glass and to consider the densification of it. This unit cell has to be chosen so that it reflects the sintering of the porous glass as a whole realistically.

The glass flow appears to be highly viscous, incompressible and Newtonian: the Stokes' creeping flow equations hold. The motion of the fluid surface is obtained from the Lagrangian representation for the velocity by considering the surface points as material points, i.e. a kinematic constraint is used. In the last few years a lot of work has been done in simulating the sintering of two-dimensional fluid regions. By now the evolution of some particular geometries can be solved even analytically, in particular using conformal mapping techniques, cf. Hopper [1]-[3]. The numerical simulation of viscous sintering is performed by successively solving the Stokes problem and employing a time step to predict the next level geometry.

The sintering of an infinite line of cylinders was simulated by Ross et al. [4]; they applied a Finite Element Method (FEM). Kuiken [5] considered domains with a rather moderately varying curvature. He used an integral representation based on the stream function and vorticity function and solved the resulting equations by employing a Boundary Element Method (BEM). In earlier work, cf. Van de Vorst et al. [6]-[10], we reported about the solution of the problem for arbitrarily shaped two-dimensional fluid regions. In those papers, the Stokes problem is described by an integral formulation based on boundary distributions of single- and double layer hydrodynamical potentials.

The next step is the simulation of three-dimensional sintering geometries. The most logical fluid regions to start with are axisymmetric geometries: bodies which are formed by rotating a two-dimensional plane around a given axis. Because of this rotational symmetry, the problem can be reduced to any plane through this particular axis, i.e. actually a two-dimensional problem has to be solved.

Jagota and Dawson [11]-[12] were the first to perform the numerical simulation of some particular axisymmetric problems. They considered the sintering of both the coalescence of two equal spheres and an infinite line of equal spheres. These simulations were carried out by applying a Finite Element Method (FEM) to solve the Stokes problem. The geometry at a next 
time step was found by using a simple forward Euler scheme.

In this paper, we present a numerical method that is capable of simulating arbitrary simply connected axisymmetric fluid regions. The numerical algorithm is based on the two-dimensional code developed by us earlier, cf. Van de Vorst et al. [6]-[10]. The BEM is applied to solve the governing Stokes equations for a fixed domain. After solving the Stokes problem, the time stepping is carried out by a more sophisticated time integrator: a variable step, variable order Backward Differences Formulae (BDF) scheme.

In section 2 of this paper we formulate the problem that has to be solved. Moreover, we briefly outline the derivation of the integral equation for the axisymmetric case. In section 3 , we highlight some details of the numerical implementation; especially some aspects of the assembling of the system of equations, the discretization of the surface curvature and the time integration. Afterwards, we will demonstrate the obtained numerical algorithm for any type of axisymmetric problem and discuss some of the evolution differences between two-dimensional and axisymmetric sintering problems. Especially we focus on the coalescence of two equal spheres which is commonly treated as unit problem in sintering literature.

\section{Problem Formulation and Solution}

The material transport by viscous sintering is modelled as a viscous incompressible Newtonian fluid driven solely by surface tension, cf. Kuiken [5]. So the Stokes creeping flow equations are valid, which read in dimensionless form

$$
\triangle \mathbf{v}-\operatorname{grad} p=0
$$

with the continuity equation

$$
\operatorname{div} \mathbf{v}=0
$$

Here $\mathbf{v}$ is the dimensionless velocity and $p$ the dimensionless pressure. The stress tensor $\mathcal{T}$ for a Newtonian fluid is defined by

$$
\mathcal{T}_{i j}=-p \delta_{i j}+\left(\frac{\partial v_{i}}{\partial x_{j}}+\frac{\partial v_{j}}{\partial x_{i}}\right)
$$

On the surface the tension, say $\mathbf{b}$, in the normal direction is proportional to the trace of the curvature tensor $\kappa_{\alpha \beta}$,

$$
\mathbf{b}:=\mathcal{T} \mathbf{n}=-\kappa_{\alpha \alpha} \mathbf{n},
$$

where $\mathbf{n}$ is the outward unit normal vector and the indices $\alpha$ and $\beta$ are varying between 1 and 2 . The equations (2.1)-(2.4) can be solved uniquely for a fixed domain up to an arbitrary rigid-body translation and rotation.

The motion of the boundary is obtained by applying the Lagrangian representation for the boundary velocity $\mathbf{v}$,

$$
\frac{\mathrm{d} \mathbf{x}}{\mathrm{d} t}=\mathbf{v}(\mathbf{x}) \quad(\mathbf{x} \in \Gamma)
$$


where $t$ is the dimensionless time. The above kinematic constraint expresses the displacement of the material boundary particles: the trajectories of those particles are followed. Hence a quasi-static approach is used to solve the viscous sintering problem.

In earlier work [6]-[10], it was shown that the BEM is ideally suited to solve two-dimensional viscous sintering problems. Therefore, it is convenient to employ the BEM for axisymmetric shapes too: hence we have to reformulate the problem in terms of an integral equation.

There are two approaches for deriving the governing integral formulation for axisymmetric problems based on hydrodynamic potentials of single- and double layers. Both methods are leading to the same equation. The first one is to obtain the integral equation by using the axisymmetric fundamental solution based on ring forces, cf. Brebbia et al. [13]. The second approach is to apply the fundamental solution derived from a point force so that a Cartesian version of the three-dimensional integral equation is obtained. Subsequently cylindrical coordinates are then substituted in this formulation. Here we will briefly summarize the latter method. More precise details of this derivation can be found for example in Lee and Leal [14], or in Becker [15] where this problem is considered as a special case of an elastostatic problem.

Let us denote a fluid region by $\Omega$ and its "smooth" surface by $\partial \Omega$. The derivation of the integral equation in the case of a Cartesian coordinate system is described in detail by, for example Pozrikidis [16]; in this specific case we obtain for a point $\mathbf{x}$ of the fluid,

$$
c_{i j} v_{j}(\mathbf{x})+\int_{\partial \Omega} q_{i j}(\mathbf{x}, \mathbf{y}) v_{j} d \partial \Omega_{y}=\int_{\partial \Omega} u_{i j}(\mathbf{x}, \mathbf{y}) b_{j} d \partial \Omega_{y}
$$

where $i$ and $j$ are varying between 1 and 3 . Here the coefficients $c_{i j}, q_{i j}$ and $u_{i j}$ are equal to respectively:

$$
\begin{gathered}
c_{i j}=\left\{\begin{array}{cl}
\delta_{i j} & \mathbf{x} \in \Omega \\
\frac{1}{2} \delta_{i j} & \mathbf{x} \in \partial \Omega,
\end{array} \quad q_{i j}(\mathbf{x}, \mathbf{y})=\frac{3\left(x_{i}-y_{i}\right)\left(x_{j}-y_{j}\right)\left(x_{k}-y_{k}\right) n_{k}}{4 \pi|\mathbf{x}-\mathbf{y}|^{5}},\right. \\
u_{i j}(\mathbf{x}, \mathbf{y})=\frac{1}{8 \pi}\left[\frac{\delta_{i j}}{|\mathbf{x}-\mathbf{y}|}+\frac{\left(x_{i}-y_{i}\right)\left(x_{j}-y_{j}\right)}{|\mathbf{x}-\mathbf{y}|^{3}}\right] .
\end{gathered}
$$

In order to obtain the integral equation for the axisymmetric case, we reformulate the representation above by employing cylindrical coordinates $(r, \theta, z)$, i.e.

$$
\mathbf{y}=\left(y_{1}, y_{2}, y_{3}\right)^{\mathrm{T}}=(\mathrm{r} \cos \theta, \mathrm{r} \sin \theta, z)^{\mathrm{T}} \text {. }
$$

Because of the rotational symmetry, we only have to determine $v_{r}$ and $v_{z}\left(v_{\theta}=0\right)$ at the intersection of the surface $\partial \Omega$ and (say) the half-space $\theta=0$. This intersection curve will be denoted by $\Gamma$; let therefore $\mathbf{x}=(R, 0, Z)^{\mathrm{T}} \in \Gamma$. After successive substitution of cylindrical coordinates and integration along the $\theta$-direction of equation (2.6) we obtain

$$
c_{\alpha \beta} v_{\beta}^{\mathrm{c}}+\int_{\Gamma} q_{\alpha \beta}^{\mathrm{c}} v_{\beta}^{\mathrm{c}} d \Gamma=\int_{\Gamma} u_{\alpha \beta}^{\mathrm{c}} b_{\beta}^{\mathrm{c}} d \Gamma
$$

were the superscript ${ }^{\mathrm{c}}$ stands for cylindrical and $\alpha, \beta$ are either 1 or 2 ; hence $\mathbf{v}^{\mathrm{c}}=\left(v_{1}^{\mathrm{c}}, v_{2}^{\mathrm{c}}\right)^{\mathrm{T}}=\left(v_{\mathrm{r}}, v_{z}\right)^{\mathrm{T}}$, etc. Moreover, the coefficients $q_{\alpha \beta}^{\mathrm{c}}$ and $u_{\alpha \beta}^{\mathrm{c}}$ depend on complete elliptic 
integrals of the first and second kind. After some analytical manipulation, the kernels on the right hand side of the integral equation above can be rewritten as

$$
\begin{aligned}
u_{\alpha \beta}^{\mathrm{e}} & =\frac{1}{8 \pi} \int_{0}^{2 \pi} \frac{A_{\alpha \beta}^{0}+A_{\alpha \beta}^{1} \cos \theta+A_{\alpha \beta}^{2} \cos ^{2} \theta}{(a-b \cos \theta)^{\frac{3}{2}}} d \theta \\
& =\frac{1}{2 \pi \sqrt{a+b}}\left[\frac{\mathrm{E}(k)}{a-b}\left(A_{\alpha \beta}^{0}+\frac{a}{b} A_{\alpha \beta}^{1}+\frac{2 a^{2}-b^{2}}{b^{2}} A_{\alpha \beta}^{2}\right)-\frac{\mathrm{K}(k)}{b}\left(A_{\alpha \beta}^{1}+\frac{2 a}{b} A_{\alpha \beta}^{2}\right)\right]
\end{aligned}
$$

where

$$
a=\mathrm{r}^{2}+R^{2}+c^{2}, \quad b=2 \mathrm{r} R, \quad c=Z-\mathrm{z}, \quad k=\sqrt{\frac{2 b}{a+b}},
$$

and the coefficients $A_{\alpha \beta}^{n}$ are given by

$$
\begin{aligned}
& A_{\alpha \beta}^{0}=-\frac{1}{2} \delta_{1 \alpha} \delta_{1 \beta} b+\delta_{1 \alpha} \delta_{2 \beta} c R-\delta_{2 \alpha} \delta_{1 \beta} c r+\left(a+c^{2}\right) \delta_{2 \alpha} \delta_{2 \beta} \\
& A_{\alpha \beta}^{1}=\left(2 a-c^{2}\right) \delta_{1 \alpha} \delta_{1 \beta}-\delta_{1 \alpha} \delta_{2 \beta} c r+\delta_{2 \alpha} \delta_{1 \beta} c R-\delta_{2 \alpha} \delta_{2 \beta} b \\
& A_{\alpha \beta}^{3}=-\frac{3}{2} \delta_{1 \alpha} \delta_{1 \beta} b .
\end{aligned}
$$

Here $\mathrm{K}(k), \mathrm{E}(k)$ are the complete elliptic integrals of the first and second kind respectively, defined by

$$
\mathrm{K}(k)=\int_{0}^{\frac{\pi}{2}} \frac{d \varphi}{\sqrt{1-k^{2} \sin ^{2} \varphi}}, \quad \mathrm{E}(k)=\int_{0}^{\frac{\pi}{2}} \sqrt{1-k^{2} \sin ^{2} \varphi} d \varphi .
$$

The kernels on the left hand side of equation (2.7) are represented by

$$
\begin{aligned}
q_{\alpha \beta}^{\mathrm{c}} & =\frac{3}{4 \pi} \int_{0}^{2 \pi} \frac{B_{\alpha \beta}^{0}+B_{\alpha \beta}^{1} \cos \theta+B_{\alpha \beta}^{2} \cos ^{2} \theta+B_{\alpha \beta}^{3} \cos ^{3} \theta}{(a-b \cos \theta)^{\frac{5}{2}}} d \theta \\
& =\frac{1}{\pi\left(a^{2}-b^{2}\right) \sqrt{a+b}}\left[\mathrm{~K}(k)\left(-B_{\alpha \beta}^{0}-\frac{a}{b} B_{\alpha \beta}^{1}+\frac{2 a^{2}-3 b^{2}}{b^{2}} B_{\alpha \beta}^{2}+\frac{a\left(8 a^{2}-9 b^{2}\right)}{b^{3}} B_{\alpha \beta}^{3}\right)\right. \\
& \left.+\frac{\mathrm{E}(k)}{a-b}\left(4 a B_{\alpha \beta}^{0}+\frac{a^{2}+3 b^{2}}{b} B_{\alpha \beta}^{1}+\frac{2 a\left(3 b^{2}-a^{2}\right)}{b^{2}} B_{\alpha \beta}^{2}-\frac{8 a^{4}-15 a^{2} b^{2}+3 b^{4}}{b^{3}} B_{\alpha \beta}^{3}\right)\right]
\end{aligned}
$$

where the coefficients $B_{\alpha \beta}^{n}$ are equal to

$$
\begin{aligned}
& B_{\alpha \beta}^{0}=-\frac{1}{2} \delta_{1 \alpha} \delta_{1 \beta} d b+\delta_{1 \alpha} \delta_{2 \beta} R d c-\delta_{2 \alpha} \delta_{1 \beta} \mathrm{r} d c+\delta_{2 \alpha} \delta_{2 \beta} d c^{2} \\
& B_{\alpha \beta}^{1}=\left(d e+B_{11}^{3}\right) \delta_{1 \alpha} \delta_{1 \beta}+\left(R^{2} n_{\mathrm{r}}-\mathrm{r} d\right) \delta_{1 \alpha} \delta_{2 \beta} c+\left(d-\mathrm{r} n_{\mathrm{r}}\right) \delta_{2 \alpha} \delta_{1 \beta} R c+\delta_{2 \alpha} \delta_{2 \beta} R c^{2} n_{\mathrm{r}}, \\
& B_{\alpha \beta}^{2}=\left(e n_{\mathrm{r}}-\mathrm{r} d\right) \delta_{1 \alpha} \delta_{1 \beta} R-\frac{1}{2} \delta_{1 \alpha} \delta_{2 \beta} b c n_{\mathrm{r}}+\delta_{2 \alpha} \delta_{1 \beta} R^{2} c n_{\mathrm{r}}, \\
& B_{\alpha \beta}^{3}=-\frac{1}{2} \delta_{1 \alpha} \delta_{1 \beta} b R n_{\mathrm{r}},
\end{aligned}
$$


and $d=-\mathrm{r} n_{\mathrm{r}}+c n_{\mathrm{z}}, e=R^{2}+\mathrm{r}^{2}$. We remark that if the point $\mathbf{x}$ is lying at the $\mathrm{z}$-axis, i.e. $R=0$, the integrals become very simple since then $b=0$ too, we obtain

$$
\begin{gathered}
u_{\alpha \beta}^{\mathrm{c}}=\frac{1}{8 \pi a^{\frac{3}{2}}} \int_{0}^{2 \pi} A_{\alpha \beta}^{0}+A_{\alpha \beta}^{1} \cos \theta+A_{\alpha \beta}^{2} \cos ^{2} \theta d \theta=\frac{1}{8 a^{\frac{3}{2}}}\left[2 A_{\alpha \beta}^{0}+A_{\alpha \beta}^{2}\right], \\
q_{\alpha \beta}^{\mathrm{c}}=\frac{3}{4 \pi a^{\frac{5}{2}}} \int_{0}^{2 \pi} B_{\alpha \beta}^{0}+B_{\alpha \beta}^{1} \cos \theta+B_{\alpha \beta}^{2} \cos ^{2} \theta+B_{\alpha \beta}^{3} \cos ^{3} \theta d \theta=\frac{3}{\pi a^{\frac{5}{2}}}\left[2 B_{\alpha \beta}^{0}+B_{\alpha \beta}^{2}\right] .
\end{gathered}
$$

The integral representation (2.7) possesses one degree of freedom only: a translation in the z-direction, say $\varphi^{\mathrm{c}}=(0,1)^{\mathrm{T}}$. There are two methods to make this integral formulation uniquely solvable for a fixed domain. One is to perform this by adding one additional variable representing this translation freedom and requiring the fluid to be stationary at a point of the $\mathrm{z}$-axis, i.e. include one extra integral constraint, cf. Hsiao et al. [17]. The other method is to "deflate" the double layer integral operator, i.e. we remove this degree of freedom as we did in the two-dimensional case, cf. Van de Vorst [8]. We prefer the latter approach since then no additional variable is required. After deflating equation (2.7) we obtain

$$
c_{\alpha \beta} v_{\beta}^{\mathrm{c}}\left(\mathbf{x}^{\mathrm{c}}\right)+\int_{\Gamma} q_{\alpha \beta}^{\mathrm{c}} v_{\beta}^{\mathrm{c}} d \Gamma+\varphi_{\alpha}^{\mathrm{c}}\left(\mathbf{x}^{\mathrm{c}}\right) \int_{\Gamma} \varphi_{\beta}^{\mathrm{c}} v_{\beta}^{\mathrm{c}} \mathrm{r} d \Gamma=\int_{\Gamma} u_{\alpha \beta}^{\mathrm{c}} b_{\beta}^{\mathrm{c}} d \Gamma
$$

This integral representation is used in the remainder of this paper.

\section{Numerical Solution}

The numerical implementation of the axisymmetric integral equation is very similar to the twodimensional case, cf. Van de Vorst et al. [6]; we actually have two unknowns $v_{r}, v_{z}$ on a boundary curve. Because of this, the mesh redistribution, which is a very important item during the simulation of a particular shape, can be performed by applying the algorithms developed for the two-dimensional case, cf. Van de Vorst and Mattheij [7]. However, the time integration method has to be modified slightly, and of course the assembling of the system of equations as well. Therefore, we will highlight some details of this numerical solution in this section.

The application of the BEM to equation (2.9) is performed by substituting linear or quadratic element-wise truncated polynomial approximations for the boundary curve, velocity and surface tension respectively, into the discretized form of (2.9). Varying the point $\mathbf{x}^{\mathfrak{c}}$ in this formulation over all the collocation points, results in the following square full rank system of linear algebraic equations, that is denoted by

$$
\mathcal{H}\left(\boldsymbol{x}^{\mathrm{c}}\right) \boldsymbol{v}^{\mathrm{c}}=\mathcal{G}\left(\boldsymbol{x}^{\mathrm{c}}\right) \boldsymbol{b}^{\mathrm{c}}\left(\boldsymbol{x}^{\mathrm{c}}\right)
$$

Here $\boldsymbol{v}^{\mathrm{c}}, \boldsymbol{b}^{\mathrm{c}}$ and $\boldsymbol{x}^{\mathrm{c}}$ are vectors that consist of the velocity, surface tension and nodal boundary coordinates respectively of all successive collocation points in the $\mathrm{r}$ - and z-direction. $\mathcal{H}$ and $\mathcal{G}$ denote the matrices obtained by element-wise integration of equation (2.9). The elliptic integrals that occur in the coefficients can be approximated by using either standard routines or a series 
representation. When the element integrals become singular, there are basically two approaches for computing them.

The first, quite commonly used, method is to express the elliptic integrals by a series expansion in terms of a logarithmic singularity, cf. Byrd and Friedman [18]. After substituting those expansions into the singular integrals, these integrals become of the same type as they occur in the two-dimensional case; so they are approximated by applying a logarithmic Gaussian quadrature formula.

The second approach is to apply the so-called Telles' transformation [19]. The goal of this method is to cancel the singularity by employing a transformation such that the Jacobian of this particular transformation is equal to zero at the singular point. Thereafter, the transformed integral can be computed using an ordinary Gaussian quadrature formula. In the case of linear elements a quadrature polynomial transformation, say $\varphi(s)=c_{0}+c_{1} s+c_{2} s^{2}$, can be constructed. The coefficients $c_{i}$ are obtained form the following three requirements,

$$
\left.\varphi^{\prime}(s)\right|_{s=\bar{s}}=0, \quad \varphi(-1)=-1, \quad \text { and } \quad \varphi(+1)=+1,
$$

where the ' denotes the derivative with respect to $s$. The first requirement enforces the Jacobian to be zero at the singular point $\bar{s}$ and both the other two requirements are needed in the Gaussian quadrature approximation. Another interesting property of this transformation is that many of the Gaussian points are distributed near the singularity. Here, we have chosen for the latter method.

When the point $\mathbf{x}^{\mathfrak{c}}$ is a point on the z-axis $(R=0)$ the elliptic integrals that occur in the coefficients disappear: the integrals become very simple as is shown by equation (2.8). In the case of linear elements, it is even possible to compute the latter element integrals analytically when they become singular.

Another feature in which the axisymmetric implementation differs from the two-dimensional case is that the coefficient matrix $c_{\alpha \beta}$ cannot be computed using rigid-body motions in the radial direction. Again there are two approaches to obtain the $(2 \times 2)$ diagonal matrices of the system of equations. The first method is to apply analytical expressions for the coefficients $c_{\alpha \beta}$, cf. Brebbia and Dominguez [20]. The second approach, which is applied here and which is extensively outlined in Becker [15], consists of two parts: the first step is using rigid-body motions in the z-direction so that the diagonal matrix coefficients that apply in this particular direction are obtained. The second step, the computation of the remaining diagonal coefficients which apply in the r-direction, is performed by employing a plane stress condition from which the velocity and tension can be computed that is valid for any arbitrary shaped region.

The next point of concern is the computation of the surface tension vector $\mathbf{b}^{c}$, i.e. the calculation of the principal surface curvature and the outer normal. Like in the two-dimensional case, a local method is used to determine the principal curvature at the discretization points on the boundary surface $\Gamma$. For example in the linear case, this curvature at a particular collocation point is approximated by fitting a quadratic polynomial through that particular point and its neighbours; say the parametric form $(\mathrm{r}(s), \mathrm{z}(s))^{\mathrm{T}}$ applies with $-1 \leq s \leq 1$. Then for the approximate principal curvature at this specific point $(s=0)$ on the curve $\Gamma$ we obtain

$$
\kappa_{m}=\frac{\mathrm{r}^{\prime} \mathrm{z}^{\prime \prime}-\mathrm{r}^{\prime \prime} \mathrm{z}^{\prime}}{\left(\left(\mathrm{r}^{\prime}\right)^{2}+\left(\mathrm{z}^{\prime}\right)^{2}\right)^{\frac{3}{2}}}+\left.\frac{\mathrm{z}^{\prime}}{\mathrm{r} \sqrt{\left(\mathrm{r}^{\prime}\right)^{2}+\left(\mathrm{z}^{\prime}\right)^{2}}}\right|_{s=0}
$$


and for the outer normal

$$
n_{\mathrm{r}}=\left.\frac{\mathrm{z}^{\prime}}{\sqrt{\left(\mathrm{r}^{\prime}\right)^{2}+\left(\mathrm{z}^{\prime}\right)^{2}}}\right|_{s=0} \quad n_{\mathrm{z}}=\left.\frac{-\mathrm{r}^{\prime}}{\sqrt{\left(\mathrm{r}^{\prime}\right)^{2}+\left(\mathrm{z}^{\prime}\right)^{2}}}\right|_{s=0} .
$$

This way we compute the surface tension at all discretization points. If a nodal point is at the symmetry axis ( $r=0)$ the second term in equation (3.2) will cancel and the curvature in this particular points is approximated using the symmetry of the problem.

After solving the system of equations (3.1) for a fixed region, a time step has to be carried out. From the kinematic motion equation (2.5), it follows, after substituting cylindrical coordinates, that the motion of the material points on the boundary curve $\Gamma$ can be described by

$$
\frac{\mathrm{d} \mathrm{r}}{\mathrm{d} t}=v_{\mathrm{r}}, \quad \frac{\mathrm{dz}}{\mathrm{d} t}=v_{\mathrm{z}}
$$

Substituting (3.3) into the algebraic equations (3.1) yields the following system of nonlinear ODEs,

$$
\frac{\mathrm{d} \boldsymbol{x}^{\mathrm{c}}}{\mathrm{d} t}=\mathcal{H}^{-1}\left(\boldsymbol{x}^{\mathrm{c}}\right) \mathcal{G}\left(\boldsymbol{x}^{\mathrm{c}}\right) \boldsymbol{b}^{\mathrm{c}}\left(\boldsymbol{x}^{\mathrm{c}}\right)
$$

In available literature about free creeping Stokes flows this system of ODEs is discretized by a simple forward Euler scheme or other explicit schemes. However, it appears that the above system of ODEs can be stiff for certain type of shapes (e.g. fluid regions which are having cusp-like regions); in such a case the time step in the forward Euler scheme has to be taken very small for obtaining a stable method. Therefore, we have implemented a variable step, variable order Backward Differences Formulae (BDF) method to solve those ODEs. More details about this implementation for the two-dimensional case are available in Van de Vorst and Mattheij [10].

\section{Numerical Results and Discussion}

In this section we show examples of the simulation of axisymmetric sintering problems. Here all fluid domains are deforming into a sphere, since this shape minimizes the outer surface that occupies a certain amount of material. The differences in evolution between two-dimensional and axisymmetric problems are discussed too.

The first example is the sintering of two equal spheres. The radius of the coalescing spheres is taken equal to 1; the initial radius of the contact circle between both spheres is set equal to 0.15 . Here, the contact radius of the coalescing circular surface will be denoted by $r$. The development during sintering of the contact radius is of physical interest. The contact radius is a measure of how "strong" a sintering compact already is. When $r$ is small, a smaller force is necessary to break the contact between both spheres than at later stages of the sintering process, when the contact radius is larger. The collocation points of the starting shape are obtained from Hopper's analytical solution for the coalescence of two equal cylinders, cf. Hopper [1]. Hence for the initial stage we assume that the geometrical differences between two touching cylinders and two touching spheres can be ignored. This is a reasonable assumption since the principal 

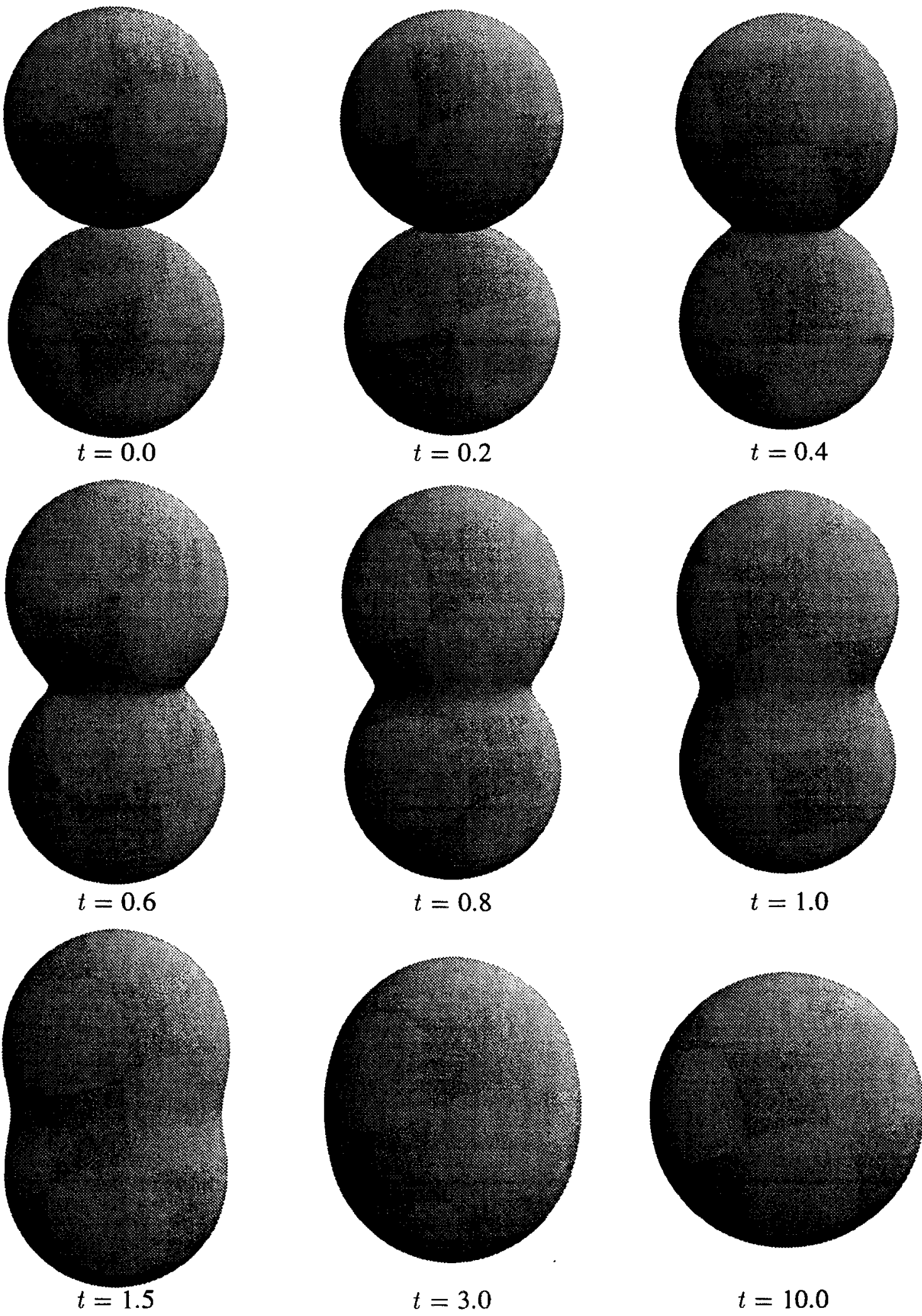

$$
t=3.0
$$

$$
t=10.0
$$

Figure 1: The coalescence of two equal spheres into one sphere occupying the same amount of fluid. 


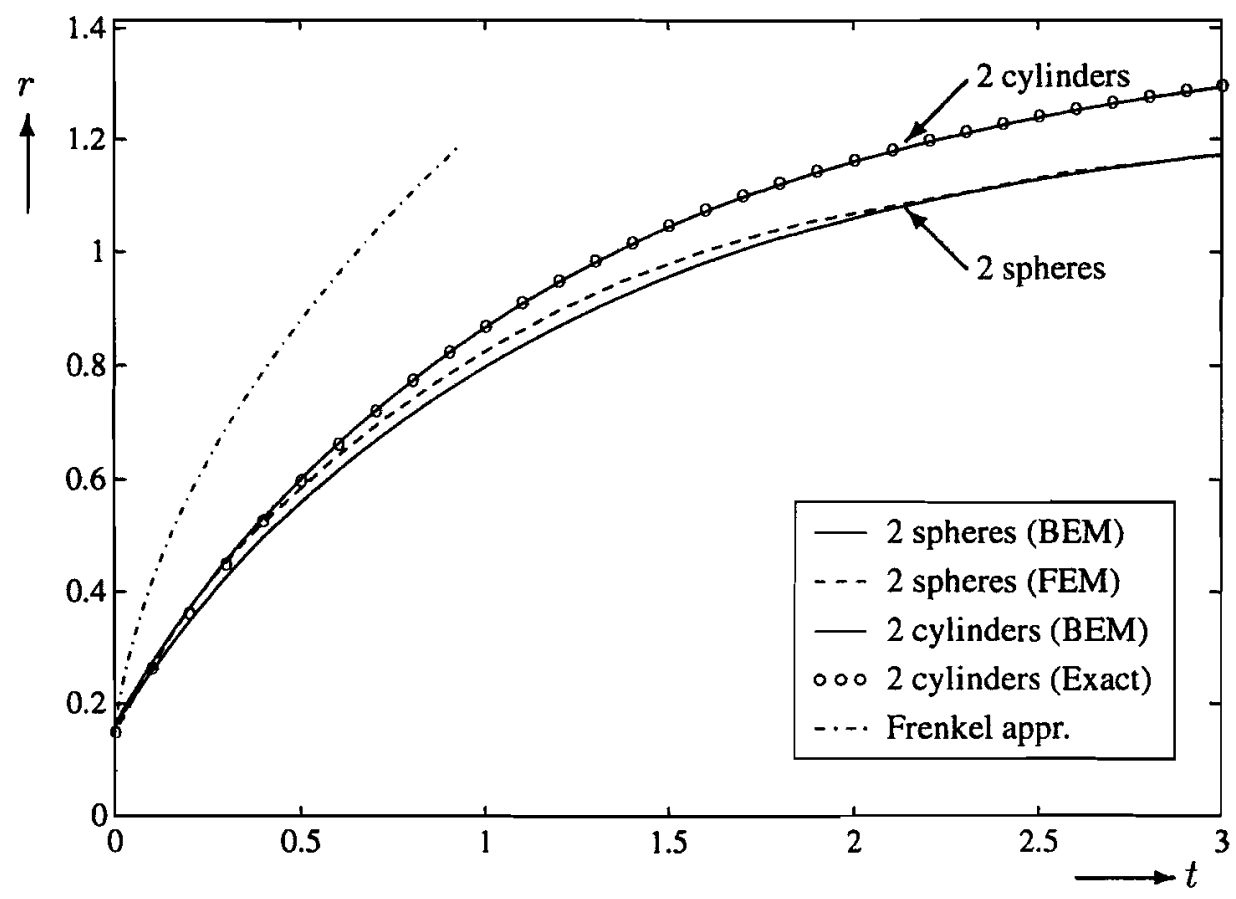

Figure 2: A comparison between the contact radius $r$ for the sintering of two equal spheres obtained by both BEM and FEM. The much faster coalescing rate derived by employing the initial stage Frenkel approximation is plotted too. The matching of Hopper's exact solution and the BEM solution is also shown when the shape is considered two-dimensional (two equal coalescing cylinders).

neck curvature in the rz-plane may assumed to be dominant (order $r^{-3}$ ) over the neck curvature in the $\theta$-direction (order $r^{-1}$ ). Only nodal points from the first quadrant of the $\theta=0$ plane are needed because of the double symmetry of the body. This symmetry is preserved during the calculations.

Figure 1 shows the simulation results of this coalescence at subsequent times $t$. In figure 2 we have plotted the numerically obtained contact radius between both spheres (solid line). The contact radius development obtained by Jagota and Dawson [11], which they derived by employing a FEM, is plotted too. In the figure, this curve is referred to as the FEM solution (dashed line). In their paper, they have printed a table of the contact surface between both spheres, called $A$, and a dimensionless time, called $\tau$. This table has to be read with care because our time normalization differs. More precisely, the following relation between their solution and ours holds,

$$
r=\sqrt{A}, \quad \text { and } \quad t=\left(\frac{3 \pi}{4}\right)^{\frac{1}{3}}\left(\tau-\tau_{0}\right)
$$

where the constant $\tau_{0}$ is added to ensure the initial $r$ to be equal to 0.15 . As can be observed from figure 2, at later time stages the BEM and FEM solutions are matching reasonably well. During the initial time stage both solutions also match, however the FEM solution follows the contact radius development of two coalescing cylinders a longer period of time. Hence, the FEM approach obtains a faster rate of coalescence for the period $0.25 \leq t \leq 2.0$. 


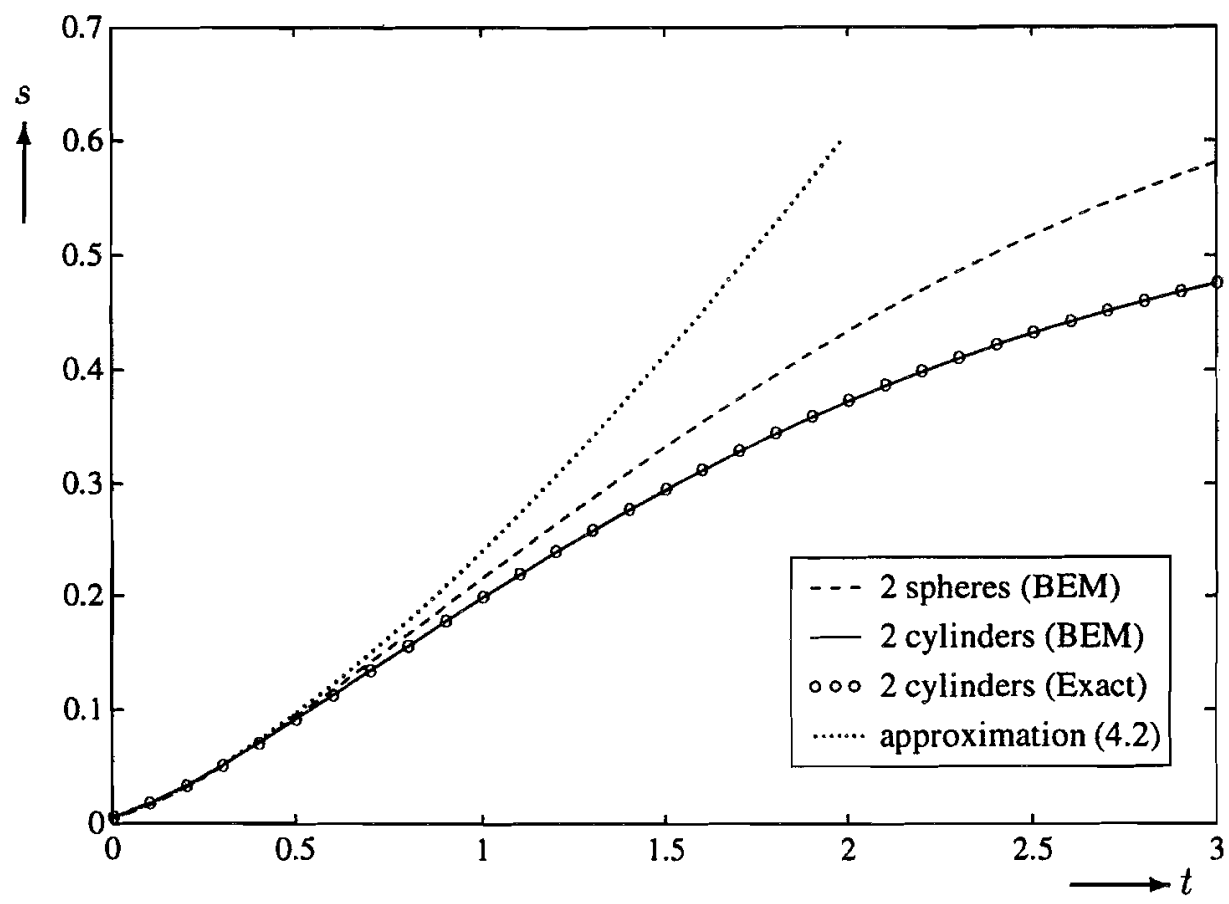

Figure 3: The shrinkage difference of the two equal spheres compared to both Hopper's exact and BEM solution of two equal coalescing cylinders. Moreover, the matching of the analytic approximate shrinkage relation with the coalescing spheres shrinkage rate is shown during the early stage.

In this figure we have plotted also the exact Hopper solution for the contact radius between two equal coalescing cylinders with initial radius 1 (circle marks), cf. Hopper [1]. The BEM-solution obtained for an axisymmetric initial geometry, considered as a two-dimensional problem, is also shown (solid line). This solution is obtained by running our 2D-code with exactly the same program parameters for the mesh generation and the BDF time integrator as in the axisymmetric case. As can be seen from the figure, both solutions are nearly identical everywhere; this gives some validation of the correctness of the axisymmetric results.

An analytical approximation for the contact radius which is commonly used in sintering literature to predict the initial stage of the coalescence is the so-called Frenkel relation, cf. Van de Vorst [9]. In figure 2 we have plotted the Frenkel relation which in the case of the considered coalescing spheres reads,

$$
t_{\mathrm{F}}=\frac{2}{3}\left(r^{2}-(0.15)^{2}\right)
$$

As can be seen (dashed dotted line), this relation predicts a much faster coalescence of the two spheres, even in the initial stage. Hence, we conclude that it does not make any sense to use this analytical relation anyway.

A more interesting feature is that Hopper's solution for two coalescing cylinders is reasonably matching with the contact radius $r$ of the two equal spheres obtained by both the FEM and BEM solution during the early stage of the coalescence. Hence during this period, the development of the contact radius of the spheres can be approximated analytically by applying Hopper's solution 


\begin{tabular}{cccccccccc}
\hline$t$ & \multicolumn{1}{c}{$\boldsymbol{s}$} & $\boldsymbol{s}$ & $t$ & $r$ & $s$ & $t$ & $r$ & $s$ \\
\hline 0.0 & 0.147767 & 0.005648 & & & & & & \\
0.1 & 0.256069 & 0.015936 & 1.1 & 0.835476 & 0.239844 & 2.1 & 1.076790 & 0.451738 \\
0.2 & 0.344573 & 0.031870 & 1.2 & 0.869421 & 0.263721 & 2.2 & 1.091422 & 0.469234 \\
0.3 & 0.423611 & 0.050464 & 1.3 & 0.900912 & 0.287162 & 2.3 & 1.104851 & 0.486204 \\
0.4 & 0.495164 & 0.070953 & 1.4 & 0.929983 & 0.310101 & 2.4 & 1.117181 & 0.502275 \\
0.5 & 0.558910 & 0.093534 & 1.5 & 0.956720 & 0.332468 & 2.5 & 1.128519 & 0.517475 \\
0.6 & 0.616099 & 0.117287 & 1.6 & 0.981281 & 0.354200 & 2.6 & 1.138930 & 0.531823 \\
0.7 & 0.668213 & 0.141559 & 1.7 & 1.003816 & 0.375236 & 2.7 & 1.148500 & 0.545299 \\
0.8 & 0.716292 & 0.166203 & 1.8 & 1.024480 & 0.395526 & 2.8 & 1.157276 & 0.558008 \\
0.9 & 0.759296 & 0.190920 & 1.9 & 1.043433 & 0.415040 & 2.9 & 1.165347 & 0.569909 \\
1.0 & 0.798940 & 0.215557 & 2.0 & 1.060821 & 0.433771 & 3.0 & 1.172759 & 0.581064 \\
& & & & & & & & \\
\hline
\end{tabular}

Table 1: Both the contact radius $r$ and shrinkage $s$ development of two coalescing spheres at subsequent time steps.

or the approximate relations that we derived in Van de Vorst [9], viz.

$$
t=\frac{\pi r}{4 \log 2-\log r} \text {. }
$$

This early stage (nearly) matching of the contact radius is not surprising since we already noted that during this coalescence stage, the principal curvature of the neck in the rz-plane is dominant over the principal curvature in the $\theta$-direction. This matching validates the use of Hopper's analytical solution for the generation of the initial axisymmetric shape.

An even more important property that can be obtained from this coalescence is shown in figure 3. In that plot we show the shrinkage rate $s$ of the coalescing spheres and compare it to the shrinking of two similarly sized cylinders. From the figure we observe that the shrinkage rate of the spheres (dashed line) is approximately linear during a large time period. Hence our calculations support the experimentally obtained linear shrinkage behaviour of regular and equally sized spherical particle packings performed by both Kuczynski [21] and Kingery and Berg [22].

Another interesting feature that can be obtained from figure 3 is that during the initial coalescence stage, the shrinkage rate is almost identical to the shrinkage of two equal cylinders. Hence the early stage shrinkage of these spheres can be approximated analytically by

$$
t \simeq \frac{4 \pi \sqrt{s}}{6 \log 2-\log s}
$$

cf. Van de Vorst [9]. The dotted line in figure 3 represents this initial stage shrinking development. As can be seen, this relation is approximately applicable for $0 \leq t \leq 1$.

Finally, in table 1 we have printed the numerically obtained values of the contact radius $r$ and the shrinkage $s$ at subsequent time points $t=0.0(0.1) 3.0$. During this particular coalescence simulation, the error which we obtained in the change of the volume was less than $1 \%$. 


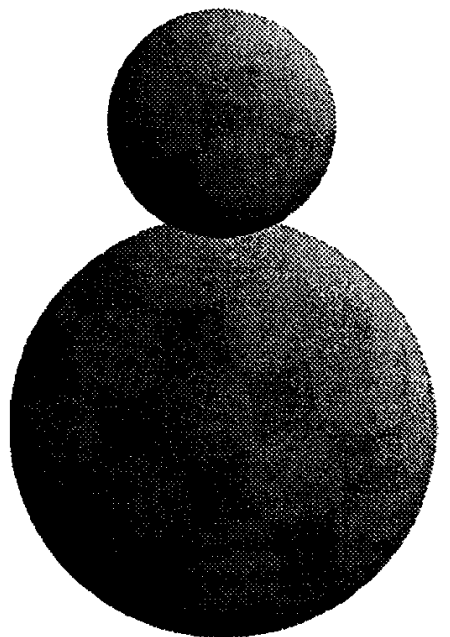

$t=0.0$

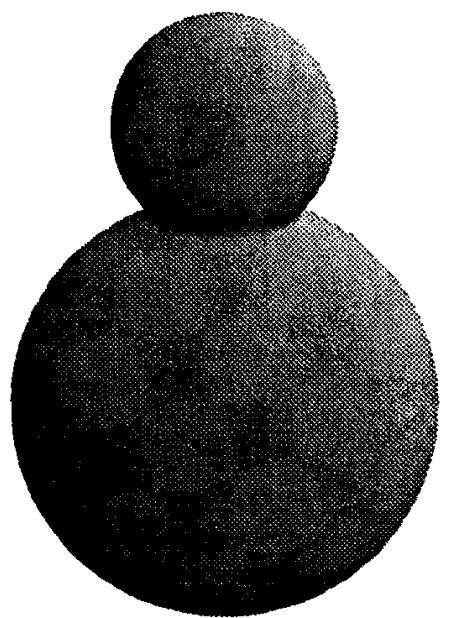

$t=0.3$

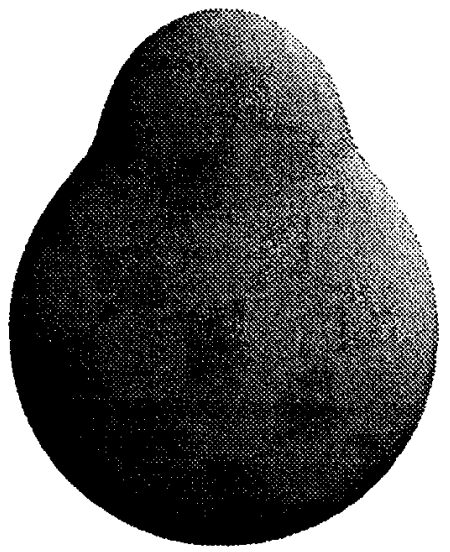

$t=1.0$

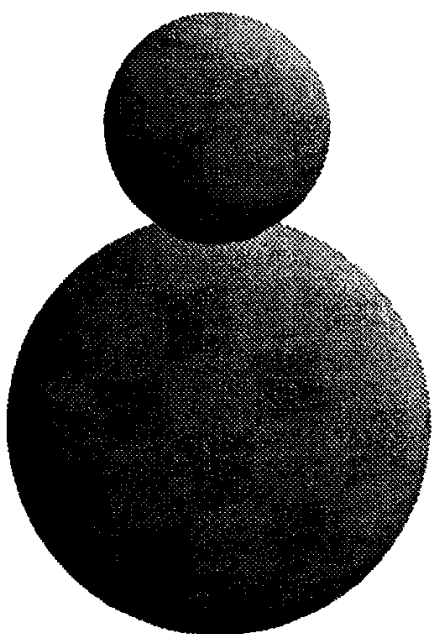

$t=0.1$

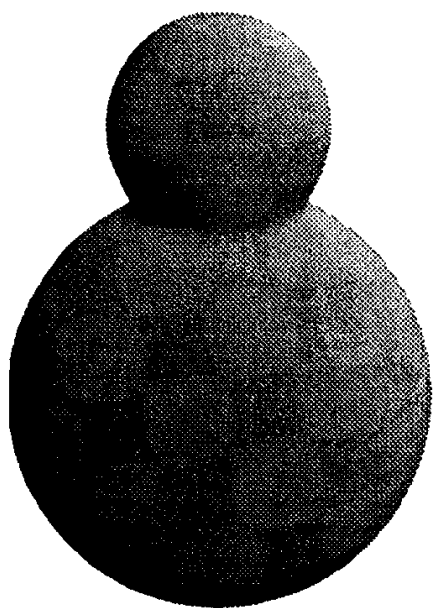

$t=0.4$

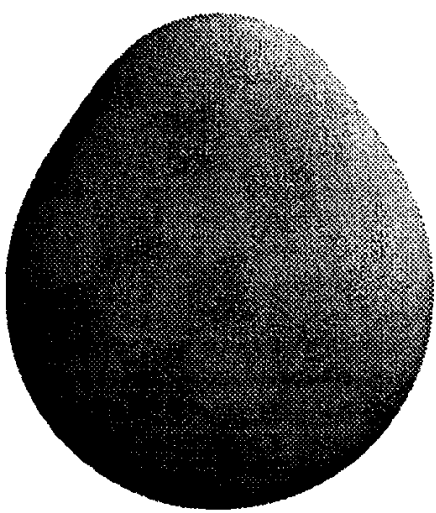

$t=1.5$

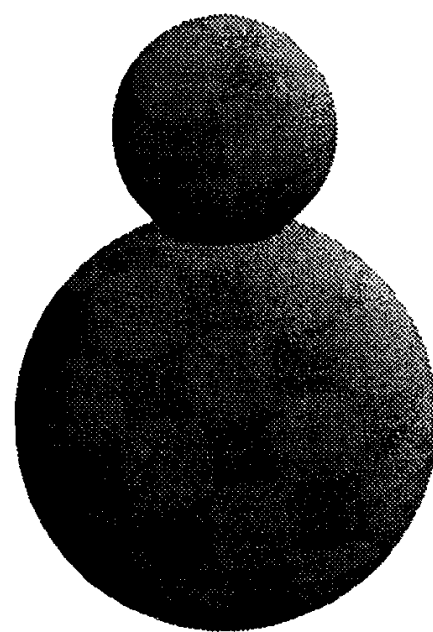

$t=0.2$

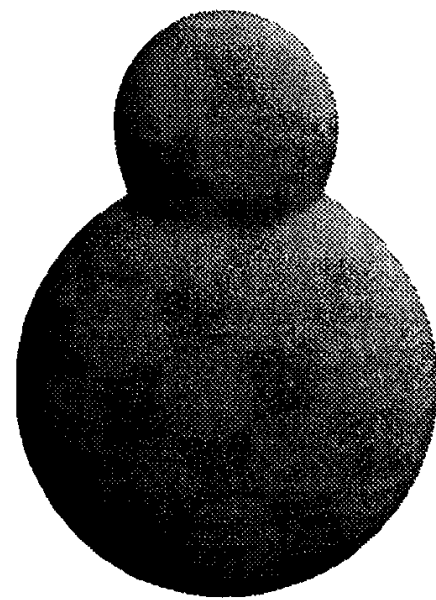

$t=0.5$

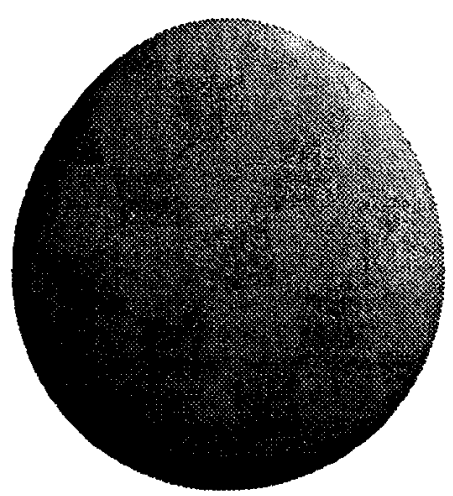

$t=2.0$

Figure 4: The coalescence of a sphere with initial radius 0.5 on a sphere with radius 1 . The smaller sphere is gradually "eaten up" by the larger one. This phenomenon is usually referred to as Ostwald ripening. 


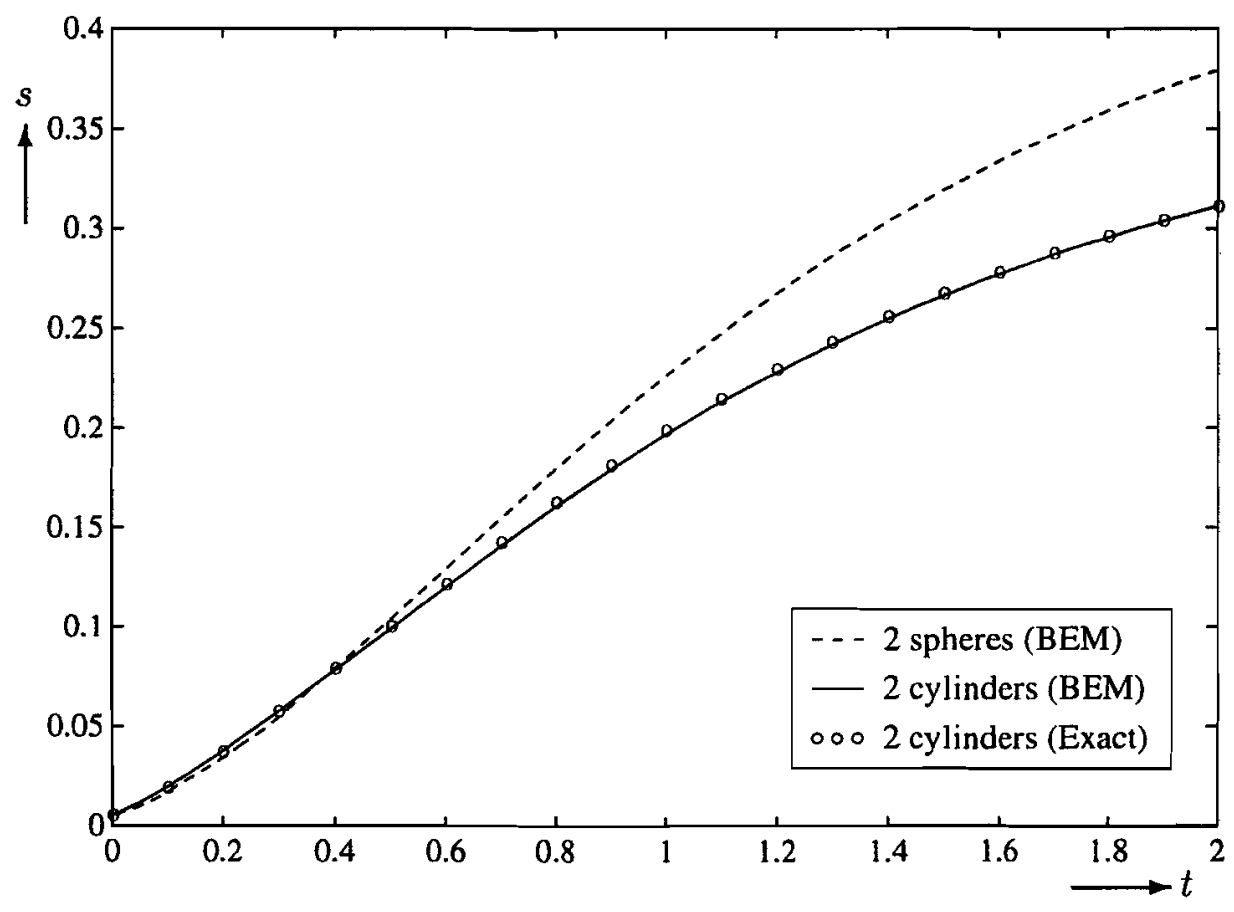

Figure 5: The comparison between the shrinkage $s$ for the sintering of the two unequal spheres and the similar two-dimensional problem shows again a matching during the early stage. Moreover, this shrinkage is behaving linearly during a large period of time.

The second axisymmetric sintering geometry we consider is the coalescence of two unequal spheres. Note that this shape has rotational symmetry only in contrast to the previous problem. Here, the initial radius of the largest sphere is taken equal to 1 and that of the smaller sphere is equal to 0.5 . The initial geometry is obtained from Richardson's analytical solution for the coalescence of two unequal cylinders, cf. Richardson [23]. The initial contact radius between both spheres is taken 0.12 .

The shape evolution is shown in figure 4. As can be observed from these pictures the smaller sphere is gradually vanishing into the larger one. This phenomenon of growth of large particles at the expense of smaller ones is usually referred to as Ostwald ripening or Cannibalism in sintering literature (cf. Mulder et al.[24]).

We observe that the early stage contact radius development between those spheres is behaving similar as compared to the two-dimensional unequal cylinders problem. Also the shrinkage rate of both problems is matching reasonably well during the early stage as can be seen from figure 5. Moreover, this figure shows that the shrinking of both spheres is evolving almost linearly as a function in time.

Next, we consider the sintering of two equal rings upon each other to compare the contact surface development of two coalescing rings, spheres and cylinders respectively. In figure 6 we have plotted the initial shape. This shape is obtained by a translation equal to 3.0 in the r-direction of the Hopper solution for two equal cylinders as considered in the first problem of this section. Hence the initial radius of these rings is equal to 1 and the width of the annular contact surface 

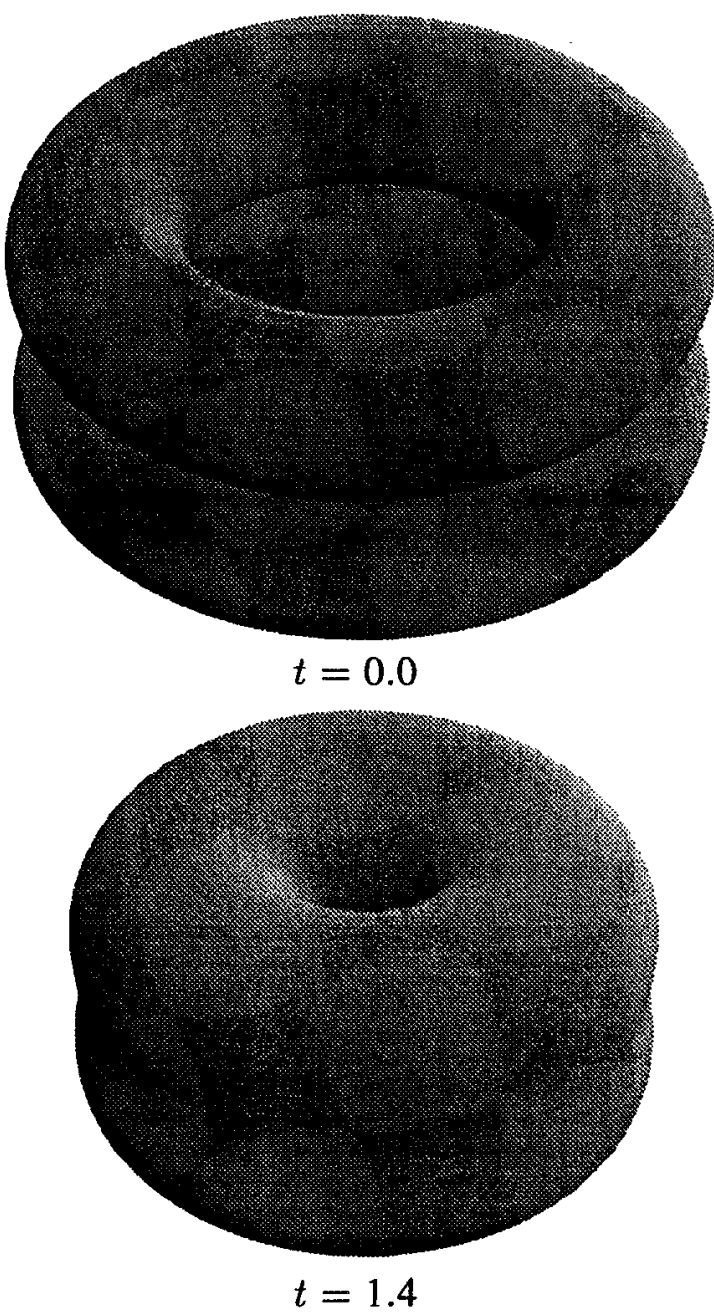

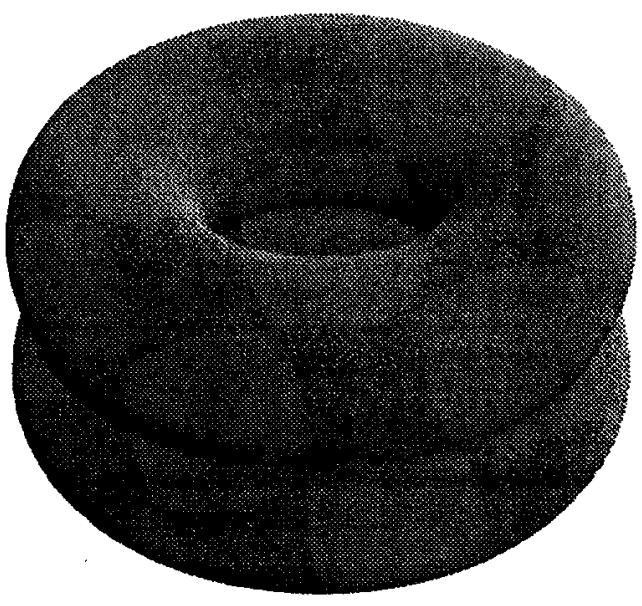

$t=0.7$

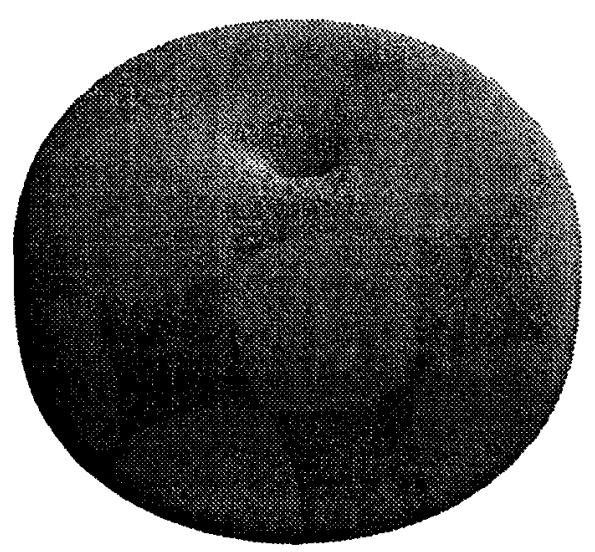

$t=2.3$

Figure 6: The coalescence of two ring-shaped viscous fluids which leads to a touching of the surface at the symmetry axis.

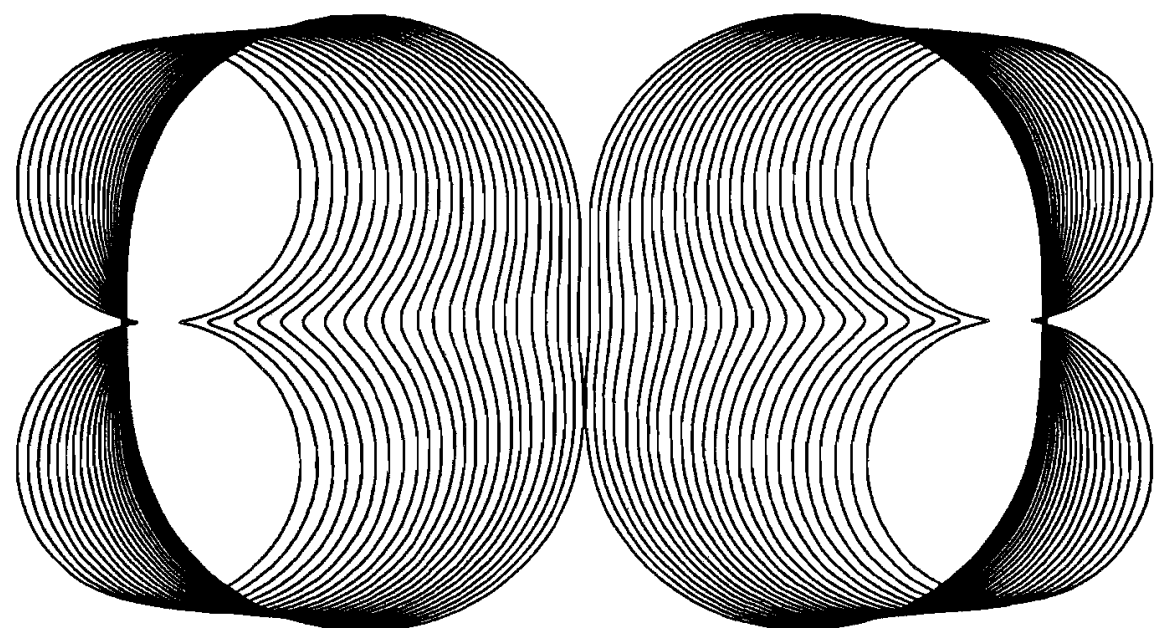

Figure 7: A cross-section of the rings in the rz-plane, $t=0.0(0.1) 2.3$. 


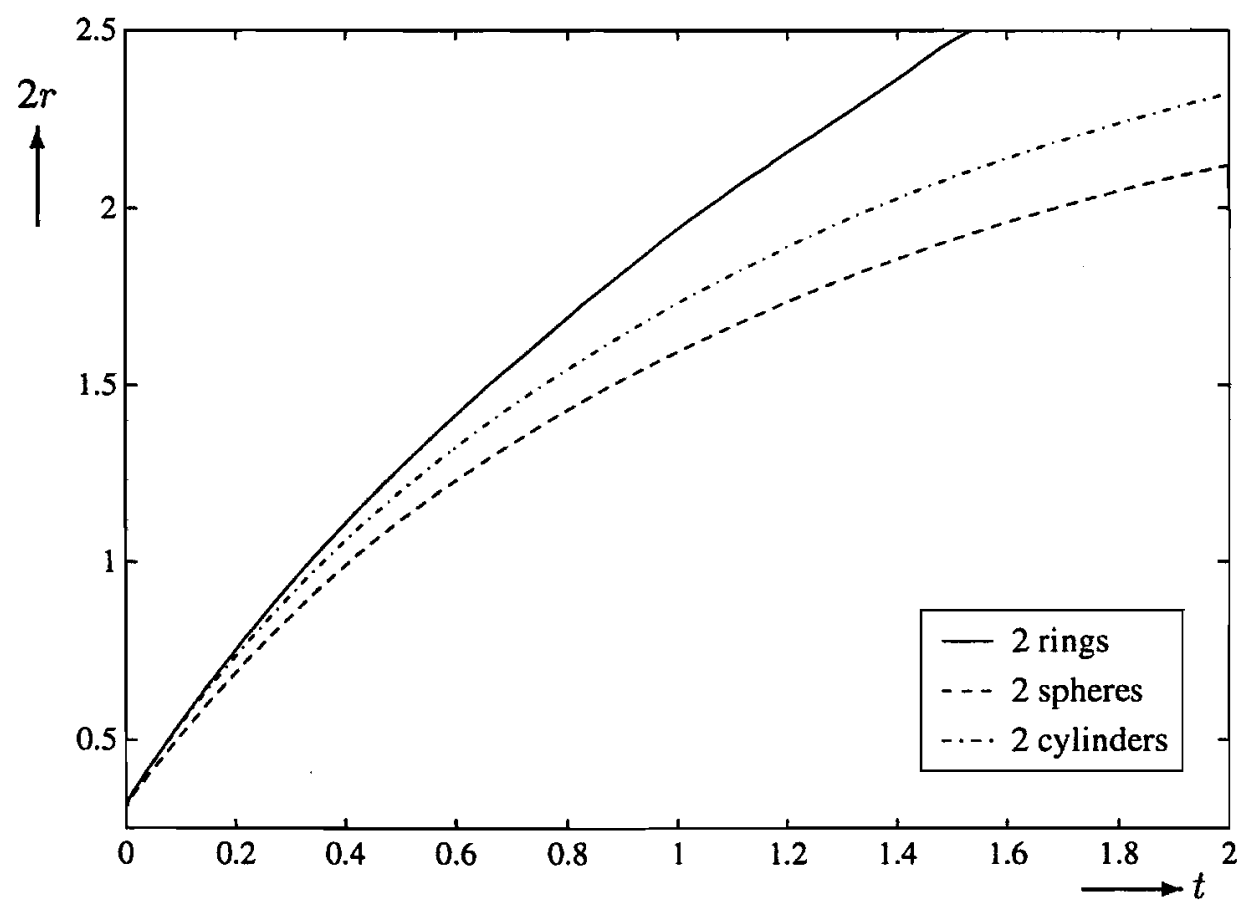

Figure 8: The matching of the width of the contact surface ( $2 r$ ) of the two equal coalescing rings, spheres and cylinders during the initial stage.

equals 0.30 (i.e. $2 r$ ).

The further deformation of the geometry is also plotted in figure 6. Moreover, a cross-section of the shape evolution at subsequent time steps of 0.1 is shown in figure 7. Besides the coalescence of both rings, it can be observed that the rings are shrinking towards the symmetry axis due to the principal curvature in the $\theta$-direction. This causes problems for our code as it is not (yet) able to handle situations where a boundary gets close to the axis of symmetry. From figure 7 we also observe that both rings do not shrink in the z-direction during the coalescence.

Since the starting geometry is similar to the coalescing spheres and cylinders considered previously, the width development of the contact surface of these three coalescence problems can be compared. Figure 8 shows those developments for each problem when time increases. Although the ring shape does not have a symmetric curvature at both sides of the annular contact surface, we see that the coalescence proceeds almost identically to the cylinder problem during the early stage. Moreover, the coalescence rate of the two spheres is behaving roughly the same during this stage too. At later time stages, the coalescence rate of the rings develops much faster compared to both other problems. This is due to the principal curvature in the $\theta$-direction, since that curvature becomes dominant over the principal curvature in the rz-plane.

Another geometry to study asymmetric neck growth is the sintering of a sphere onto a ring. This is a straightforward axisymmetric generalization of the two-dimensional sintering of three circles with an angle between the centres of those circles, cf. Van de Vorst [9]. The interesting aspect of this geometry is that if the initially chosen top angle of the cone formed by the centre of the sphere and the centre circle of the ring, is small, an internal hole can occur during sintering. 


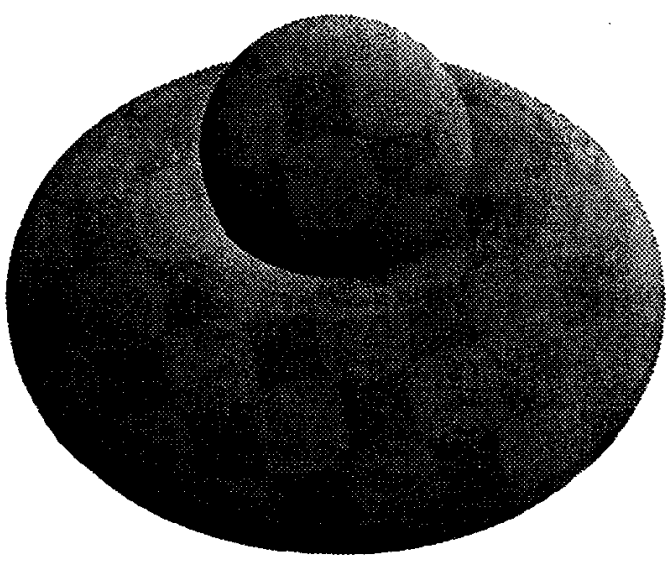

$t=0.0$ (above view)

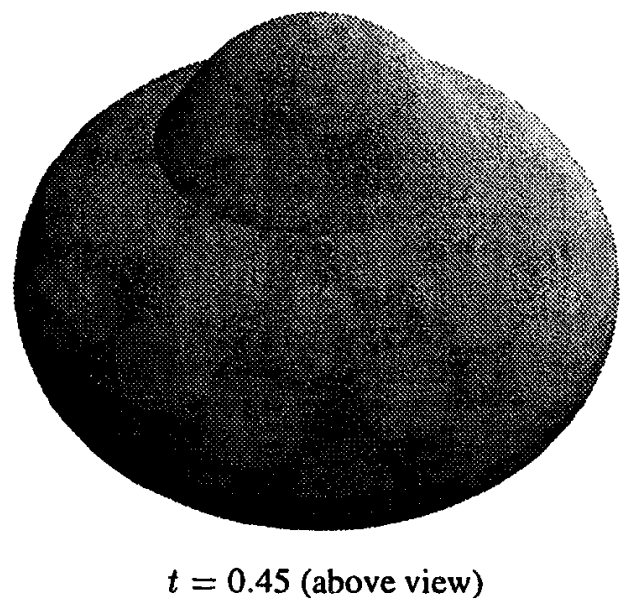

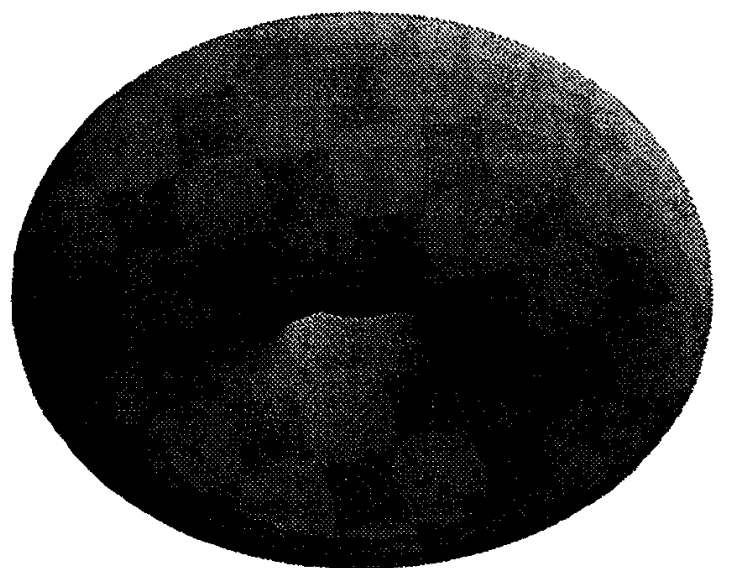

$t=0.0$ (below view)

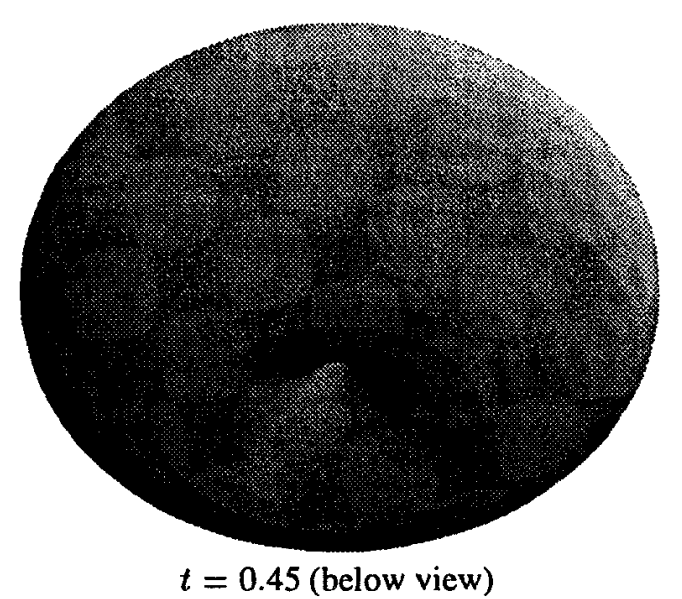

Figure 9: The coalescence of a sphere on a ring-shaped viscous fluid to study asymmetric neck growth.

This hole development also depends on the initial contact surface width between the sphere and the ring.

The first picture of figure 9 shows the initial shape of such a system. The geometry is viewed from above. In this particular case, the angle between the centre of the sphere and the ring centre line is taken equal to $100^{\circ}$ when considered a cross-section in the rz-plane. From this picture, it is clear that when the angle is large, no internal holes during sintering will occur, since the larger the angle between sphere and ring, the deeper the sphere is initially placed inside the ring. Note, that for different angles we obtain different ring sizes. The initial radius of both the sphere and the ring is set equal to 0.5 , and the contact surface is again obtained by applying Hopper's analytical solution for two equal spheres; here the contact radius is taken equal to 0.1 , thus the contact surface width is equal to 0.2 .

The other picture of figure 9 is taken at time $t=0.45$. At that moment, the inside part of the ring is nearly touching the symmetry axis: an internal hole is formed. In order to obtain a more detailed view of this hole formation, we consider figure 10 . Here, the evolution of the cross-section of the shape in the rz-plane is plotted at subsequent time steps. Figure $10^{\mathrm{a}}$ is 


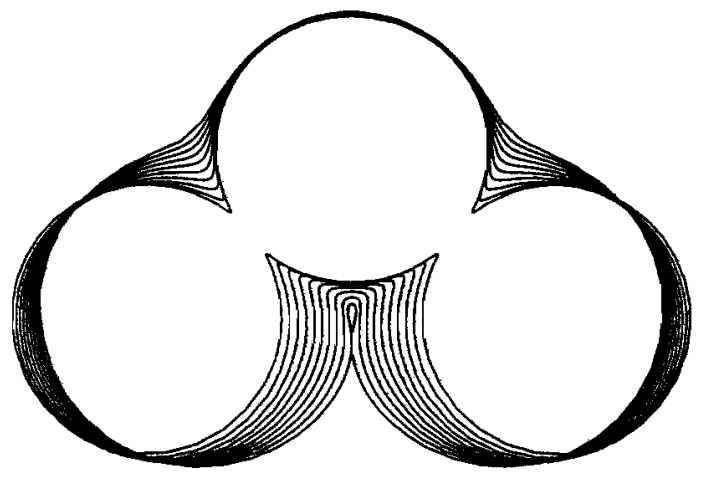

a. The initial angle is $100^{\circ}$ and the contact surface width is equal to 0.20 . The curves refer to $t=$ $0.0(0.05) 0.45$.

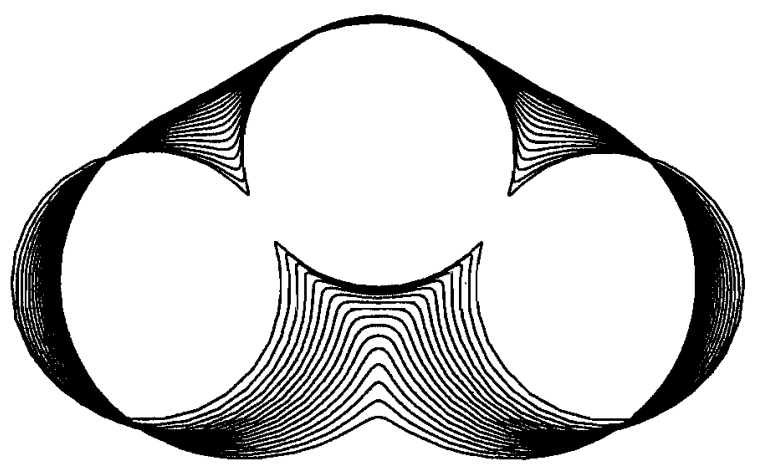

c. The initial angle is $120^{\circ}$ and the contact surface width is equal to 0.20 . The curves refer to $t=0.0(0.05) 0.8$.

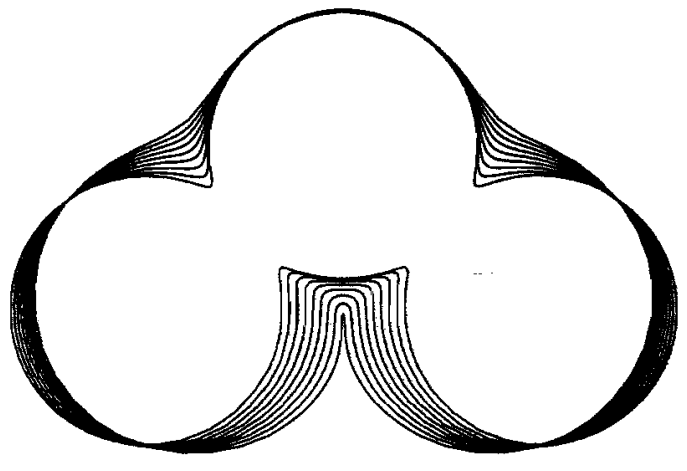

b. The initial angle is $100^{\circ}$ and the contact surface width is equal to 0.40 . The curves refer to $t=0.0(0.05) 0.4$

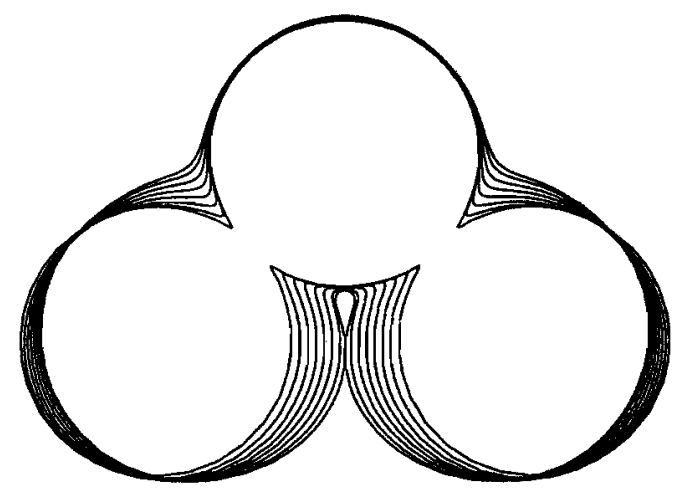

d. The initial angle is $90^{\circ}$ and the contact surface width is equal to 0.20 . The curves refer to $t=0.0(0.05) 0.3$ and $t=0.338$.

Figure 10: A cross-section of the coalescing sphere and ring in the rz-plane during sintering. The differences in evolution are shown when both the angle and/or the contact surface width between the sphere and ring are varied.

showing the sintering of the above described geometry at times $t=0.0(0.05) 0.45$. At the final time step a hole occurs: the remaining inner parts of the ring are nearly touching the symmetry axis.

Furthermore, we will discuss the differences in the evolution when some characteristics of the shape are slightly changed. In figure $10^{\mathrm{b}}$ we have plotted the evolution of the same geometry, but now the initial contact surface width is taken two times larger, i.e. 0.4 . As can be seen, no internal hole will develop since the boundary is almost completely touching the symmetry axis: a line contact occurs.

Figure $10^{c}$ shows the effect of an increase of the initial angle between the sphere and ring. The angle is set equal to $120^{\circ}$ and the contact surface width is again 0.2 . The curves refer to values of time $t=0.0(0.05) 0.8$. When comparing this simulation to the results of figure $10^{\mathrm{a}}$ we observe that no internal hole is found. Now, the development of a cusp at this particular part is 
the only effect. This plot also shows that the cusp smooths out as time increases. It appears that the cusp is smoother if the initial contact surface width is larger too, i.e. a similar behaviour as we found for the shape of figure $10^{\mathrm{b}}$.

The effect of decreasing the angle between the sphere and the ring is shown in figure $10^{\mathrm{d}}$. Here, the angle is set equal to $90^{\circ}$. Again an internal hole is formed and this hole is larger than the one in figure $10^{\mathrm{a}}$. Hence the shape is behaving like we would expect.

If we model and simulate the geometries of figure 10 as two-dimensional fluids, it appears that such a hole development does not take place for all those shapes. A hole occurring only if the angle is taken much smaller, thus when the two outer cylinders are almost touching initially.

Finally, we simulate the rounding of two opposite cones that are pressed together. From a two-dimensional point of view, the initial shapes are rhombic geometries. The largest diagonal line inside those shapes is taken equal to 2 and the angle of the lines that define the sharpest corners is initially set equal to 15,30 and 60 degrees respectively. In figure 11 the rounding is shown of the cone with initial peak of $15^{\circ}$. The cross-section of the deformed shapes are plotted in figure 12 at subsequent times $t=0.0(0.025) 0.5$. A remarkable phenomenon that can be observed form these pictures is that the cones deform inside the prescribed initial geometry of the sharpest corner parts. Hence, no fluid blob occurs at the top of the cones when they are deforming. It can also be seen that the rounding is proceeding slower by increasing angle.

\section{Conclusion}

The examples of section 4 demonstrate both the capabilities and limitations of our numerical code. It is shown that the shrinkage of both two equal and two unequal spheres is proceeding at a linear rate during the initial stage. This shrinkage rate can be approximated analytically by applying the corresponding two-dimensional analytic solutions of these problems. Moreover, the rounding of sharp peaks is developing like the corresponding two-dimensional problems. Asymmetric neck growth is studied by considering the coalescence of two equal rings and a sphere on a ring. These simulations show the influence due to the principal curvature in the azimuthal direction on the shape deformation. These latter examples also demonstrate both the touching of boundaries and the formation of inner holes in the three-dimensional case.

It is clear that a further improvement of the simulation capabilities may be achieved by the design of a detect and handle algorithm for the case of a touching boundary. This problem is not quite straightforward, since a measure has to be introduced that indicates when two boundaries are touching. Of course, this measure has to depend on the discretization error too. After the touching, the outer surface curvature of the coalescing region has to be prescribed. We think that a most sensible value for this curvature may be obtained from the unit problem of the sintering of two spheres. The initial radii of those spheres should then be chosen such that they mimic the both parts of the coalescing surface locally quite well. And of course, experimental verification has to be carried out, to justify the model chosen. 

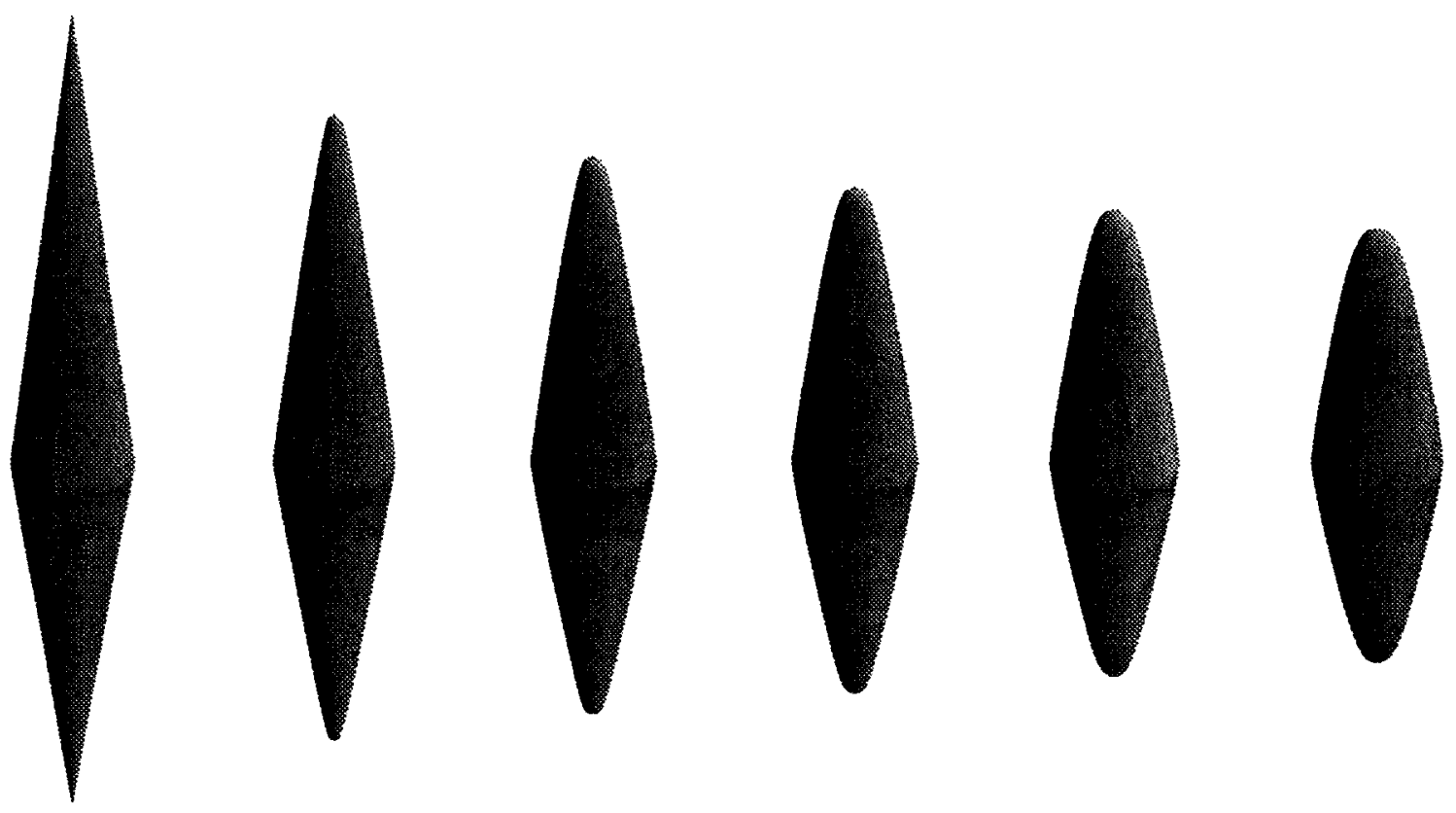

$t=0.00$

$t=0.02$

$t=0.04$

$t=0.06$

$t=0.08$

$t=0.10$

Figure 11: The rounding of two opposite cones that are pressed together. The initial angle of the initial peak equal to $15^{\circ}$

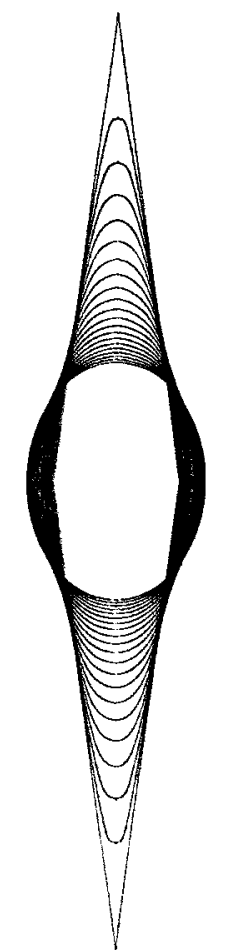

peak $15^{\circ}$

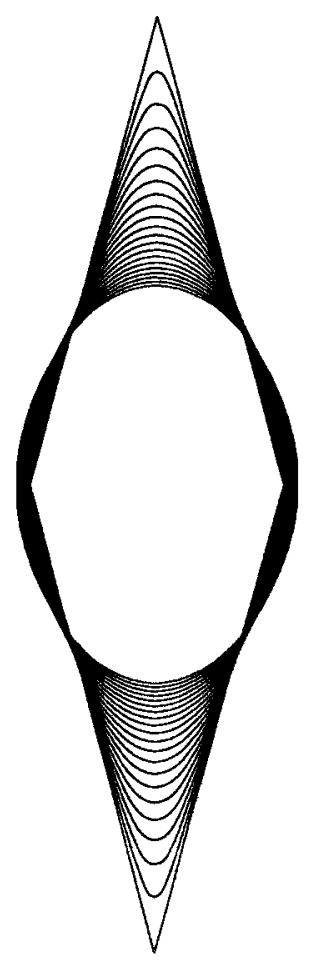

peak $30^{\circ}$

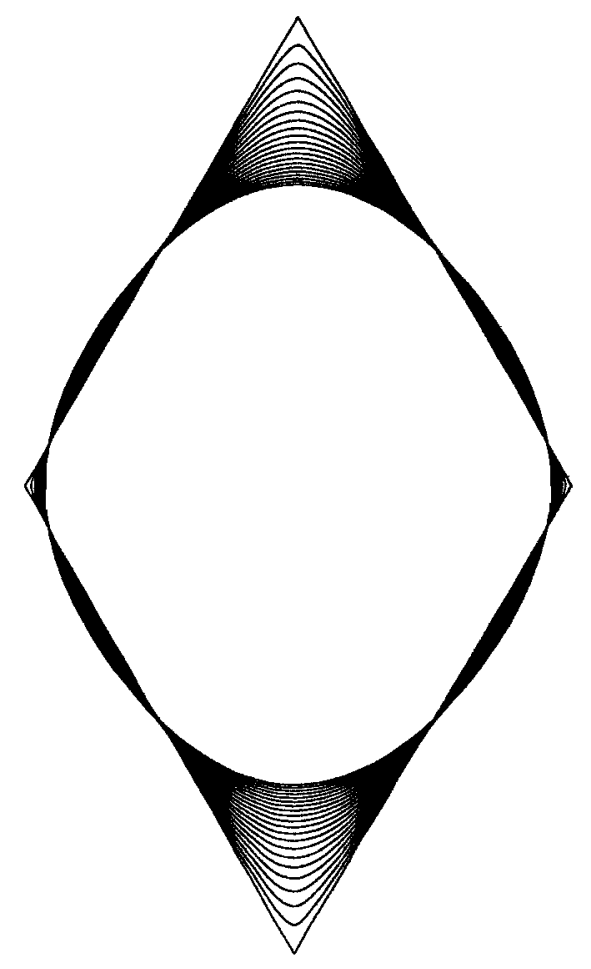

peak $60^{\circ}$

Figure 12: The cross-section of the rounding of two opposite cones that are pressed together at $t=0.0(0.025) 0.5$. The initial angle of the peaks are taken equal to 15,30 and 60 degrees respectively. The cones deform inside the prescribed initial geometry of the peaks and the rounding is proceeding slower by increasing angle. 


\section{Acknowledgement}

This research was supported by the Technology Foundation (STW).

\section{References}

[1] Hopper, R.W., Plane Stokes flow driven by capillarity on a free surface, J. Fluid Mech., 1990, 213, 349-375.

[2] Hopper, R.W., Plane Stokes Flow driven by capillarity on a free surface, 2: Further Developments, J. Fluid Mech., 1991, 230, 355-364.

[3] Hopper, R.W., Stokes Flow of a Cylinder and Half-Space Driven by Capillarity, J. Fluid Mech., 1992, 243, 171-181.

[4] Ross, J.W., Miller, W.A. \& Weatherly, G.C., Dynamic Computer Simulation of Viscous Flow Sintering Kinetics, J. Appl. Phys., 1981, 52, 3884-3888.

[5] Kuiken, H.K., Viscous sintering: the surface-tension-driven flow of a liquid form under the influence of curvature gradients at its surface, J. Fluid Mech., 1990, 214, 503-515.

[6] Van de Vorst, G.A.L., Mattheij, R.M.M. \& Kuiken, H.K., A Boundary Element Solution for Two-Dimensional Viscous Sintering, J. Comput. Phys., 1992, 100, 50-63.

[7] Van de Vorst, G.A.L. \& Mattheij, R.M.M., Numerical Analysis of a 2-D Viscous Sintering Problem with Non Smooth Boundaries, Computing 49, 239-263, 1992.

[8] Van de Vorst, G.A.L., Integral Method for a Two-Dimensional Stokes Flow with Shrinking Holes Applied to Viscous Sintering, J. Fluid Mech.,1993, 257, 667-689.

[9] Van de Vorst, G.A.L., Modelling and Numerical Simulation of Viscous Sintering, Ph.D. Thesis, Eindhoven University of Technology, 1994.

[10] Van de Vorst, G.A.L. \& Mattheij, R.M.M., A BEM-BDF Scheme for Curvature Driven Moving Stokes Flows, J. Comput. Phys., 1994. (Submitted).

[11] Jagota, A. \& Dawson, P.R., Micromechanical Modeling of Powder Compacts-I. Unit Problems for Sintering and Traction-Induced Deformation, Acta. Metall., 1988, 36, 2551-2561.

[12] Jagota, A. \& Dawson, P.R., Simulation of the Viscous Sintering of Two Particles, J. Am. Ceram. Soc., 1990, 73, 173-177.

[13] Brebbia, C.A., Telles, J.C.F. \& Wrobel, L.C., Boundary Element Techniques, SpringerVerlag, Berlin, 1984. 
[14] Lee, S.H. \& Leal, L.G., The Motion of a Sphere in the Presence of a Deformable Interface. Part 2: Numerical study of the translation of a sphere normal to an interface, J. Colloid Interface Sci., 1982, 87, 81-106.

[15] Becker, A.A., The Boundary Element Method in Engineering, McGraw-Hill Book Company, London, 1992.

[16] Pozrikidis, C., Boundary Integral and Singularity Methods for Linearized Viscous Flow. Cambridge University Press, Cambridge, 1992.

[17] Hsiao, G.C., Kopp, P. \& Wendland, W.L., Some applications of a Galerkin-collocation method for boundary integral equations of the first kind, Math. Meth. in the Appl. Sci., $1984,6,280-325$.

[18] Byrd, P.E. \& Friedman, M.D., Handbook of Elliptic Integrals for Engineers and Scientists. Springer-Verlag, Berlin, 1971.

[19] Telles, J.C.F., A Self-adaptive coordinate Transformation for Efficient Numerical Evaluation of General Boundary Element Integrals, Int. J. Num. Meth. in Engng., 1989, 24, 959-973.

[20] Brebbia, C.A. \& Dominguez, J., Boundary Elements: An Introductory Course, Computational Mechanics Publications, Southampton, 1989.

[21] Kuczynski, G.C., Study of the Sintering of Glass, J. Appl. Phys., 1949, 20, 1160-1163.

[22] Kingery, W.D. \& Berg, M., Study of the Initial Stages of Sintering by Viscous Flow, Evaporation-Condensation, and Self-Diffusion, J. Appl. Phys., 1955, 26, 1205-1212.

[23] Richardson, S., Two-dimensional slow viscous flows with time-dependent free boundaries driven by surface tension, Euro. J. App. Math., 1992, 3, 193-207.

[24] Mulder, C.A.M., Van Lierop, J.G. \& Frens, G., Densification of $\mathrm{SiO}_{2}-$ Xerogels to glass by Ostwald ripening, J. Non-Cryst. Solids, 1986, 82, 92-96. 